\title{
Comparative Residual Stress Evaluation in SLM 3D-printed Al- Si-Mg alloy (RS-300) using the Contour Method, Hole Drilling Laser Speckle Interferometry, X-ray Diffraction and Xe pFIB- DIC micro-ring-core milling
}

\author{
Eugene S. Statnik ${ }^{1, *}$, Fatih Uzun ${ }^{2}$, Svetlana A. Lipovskikh ${ }^{1}$, Sviatoslav I. Eleonsky ${ }^{3}$, Vladimir S. Pisarev ${ }^{3}$, Pavel \\ A. Somov ${ }^{1}$, Alexey I. Salimon ${ }^{1}$, Yuliya V. Malakhova ${ }^{4}$, Aleksandr G. Seferyan ${ }^{4}$, Dmitry K. Ryabov ${ }^{4}$ and Alexan- \\ der M. Korsunsky ${ }^{2,1}$

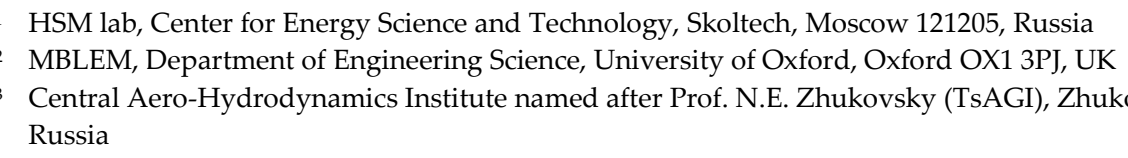

\begin{abstract}
SLM Additive Manufacturing has demonstrated great potential for aerospace applications when structural elements of individual design and/or complex shape need to be promptly supplied. 3D-printable AlSi10Mg (RS-300) alloy is widely used for the fabrication of different structures in aerospace industry. The importance of the evaluation of residual stresses that arise as a result of complex 3D-printing process thermal history is widely discussed in literature, but systematic assessment remains lacking for their magnitude, spatial distribution, and comparative analysis of different evaluation techniques. In this study we report the results of a systematic study of residual stresses in a 3D-printed double tower shaped samples using several approaches: the contour method, blind hole drilling laser speckle interferometry, X-ray diffraction, and Xe pFIB-DIC microring-core milling analysis. We show that a high level of tensile and compressive residual stresses is inherited from SLM 3D-printing and retained for longer than 6 months. The stresses vary over a significant proportion of the material yield stress. All residual stress evaluation techniques considered returned comparable values of residual stresses even regardless of dramatically different dimensional scales from millimeters for the Contour Method down, laser speckle interferometry and $\mathrm{XRD}$ and down to small fractions of a $\mathrm{mm}(70 \mu \mathrm{m})$ for Xe pFIB-DIC ring-core drilling. The use of residual stress evaluation is discussed in the context of optimizing the printing strategy to enhance the mechanical performance and long-term durability.
\end{abstract}

Keywords: SLM; Al-Si-Mg alloy; residual stress; contour measurements; laser speckle-pattern interferometry; Xe pFIB-DIC; FEniCS

\section{Introduction}

Selective Laser Melting (SLM) 3D-printing as a technology of rapid fabrication of net shape or near-net shape metal parts for various applications has matured in last 20 years and reached the stage of readiness for mass-production. In this context, the issues of quality stability and service life management become the principal focus of further development. This is especially important for $3 \mathrm{D}$-printable $\mathrm{Ti}, \mathrm{Ni}$, and $\mathrm{Al}$ alloys that recently began to see increasing use in the aerospace domain [1 3]. Fatigue fracture tolerance is known to be sensitive to microstructure variability and tensile residual stresses that persist after various fabrication operations [4]. Gas turbine engine components made of Ti and $\mathrm{Ni}$ are susceptible to fatigue linked to the flight-related thermal and mechanical cyclic loading, and to the higher frequency loading related to turbine rotation and blade 
vibration [5]. To pave the way for widespread adoption, 3D-printed parts made from these alloys must be carefully assessed in terms of fatigue durability with the same rigour as traditional materials [6].

SLM 3D-printable Al-Si-Mg alloy has been recently utilized for the fabrication of thermoregulation casing of a gamma-ray detector used in satellite applications [7]. Heatingcooling cycles are typical for many outer space structures and devices forming part of satellite equipment that alternatively pass lit and shadowed zones. These thermal excursions cause cycles of expansion and contraction that may promote fatigue cracking in 3Dprinted parts especially when tensile residual stresses are inherited after fabrication. Relaxation annealing or surface modification (shot peening or laser shock peening) are traditional approaches to controlling the residual stresses. Both techniques have some drawbacks: annealing may cause intolerable distortions that require subsequent machining that brings additional costs and new residual stresses in the surface layers, while surface modification may increase roughness and give rise to significant residual stresses in the nontreated core regions.

Repeated localized laser melting that leads to the fusion of metal powder particles followed by cooling are the fundamental physical mechanisms involved in the SLM 3Dprinting. The complexity of thermal history of a certain material volume becomes apparent if many important phenomena are considered, e.g. the completeness of homogenization in the liquid phase; phase separation for alloys with solid solution phase and associated liquation and localized oversaturation of solid solutions; grain nucleation and growth from overcooled liquids; shrinkage at solidification; aging of solid solutions and many others. High-temperature gradients and steep cooling rates during building provoke diverse structure formation and variation processes that ultimately give rise to nonuniform plastic deformation and the associated permanent inelastic strains (eigenstrains) that act as the origins of residual stresses [8].

The quantitative evaluation of residual stresses in SLM 3D-printed metallic materials came into the focus of research interest since the first decade of the 21st century [9] that paved the way for new techniques based on X-ray scattering and various sectioning-based methods at the macro- to micro- to nano-scale. Currently dozens of publications on this subject are published annually, including highly cited comprehensive overviews [10]. Steels of the stainless and maraging varieties, Ti-6Al-4V and $\mathrm{Ni}$ alloys (Inconels and Invars) are leading in terms of the number of publications devoted to residual stresses in SLM 3D-printed metals. Nevertheless, a significant number of reports [11 20] were published recently, aiming to elucidate the different aspects of residual stresses in SLM-printable Al-Si-Mg alloys.

To the best of the authors' knowledge, the first article on residual stresses in a SLM 3D-printed aluminium alloy (AlSi10Mg) [11] was published in 2018, reporting the use of the X-ray diffraction method for experimental measurements. Tensile and compressive stresses as high as $100 \mathrm{MPa}$ were found in 3D-printed thin $(t=1.2 \mathrm{~mm})$ sheet part of complex curveted shape. Generally speaking, laboratory X-ray diffraction remains the dominant method for experimental evaluation of residual stresses due to its non-destructive nature, and in spite of the numerous known disadvantages such as the shallow region of interaction and large lateral averaging area. Other non-destructive (e.g., precise external shape measurements by means of contact and contactless scanning) and destructive techniques (like hole drilling) were applied and reported. The interpreting and modelling of residual stresses commonly require various computational data processing approaches and computer simulations.

The FIB-DIC micro-ring-core drilling method is the extension of the hole drilling method towards the microscopic dimensional scale. The method has been a developing project since the middle of 2000s [21]. At the present day this method gives unprecedented opportunities to correlate the structural peculiarities and residual stresses and is clearly relevant for 3D-printed materials. FIB-DIC micro-ring-core drilling method suggests the use of relatively complex dual FIB-SEM microscopes and skilled operators, but the robustness of the measurements has already reached the pre-standardization stage [22]. There 
are very few reports of using FIB ring-core drilling method to measure residual stresses in SLM 3D-printed materials [17] and no reports on SLM 3D-printed Al-alloys have been found in the literature to date. One of the reasons as believed is the scale factor - the correspondence of the drilled ring diameter and average grain size is very important for FIB ring-core drilling method. In order to probe Type I stresses (equivalent to engineering scale residual stresses) the diameter of a ring must be set to several grain sizes - a condition that is hard to satisfy with the most popular Ga FIB-SEM microscopes for large and medium-size grains (20 $\mathrm{mm}$ and bigger) in materials such as Al-Si-Mg printable alloys.

The present authors recently studied the peculiarities of grain structure and mechanical performance of 3D-printed RS-333 Al-alloy parts $[23,24]$ and considered the possible influence of the residual stresses on the variation of mechanical response. In the present article, we report a systematic comparative study of under-surface residual stresses in 3Dprinted RS-300 series (AlSi10Mg) alloy samples using a number of macroscopic destructive techniques such as the Contour Method, hole drilling coupled with laser speckle interferometry, and microscopic Xe-ion pFIB-DIC ring-core drilling. Conventional X-ray diffraction $\left(\sin ^{2} \psi\right)$ measurements were also carried out to complete the multi-technique characterization study.

It has been experimentally found that the high levels of tensile and compressive residual stresses inherited after SLM 3D-printing vary in the range $40 \ldots 200 \mathrm{MPa}$ (i.e. up to the yield stress) and remain unrelaxed for at least 6 months. All evaluation techniques return comparable estimates of residual stress values, in spite of the dramatically different dimensional scales - from millimeters for the contour method and the laser speckle interferometry down to $70 \mu \mathrm{m}$ for Xe pFIB-DIC ring-core drilling. In the concluding part of the manuscript, the authors address the subject of how the tuning of printing parameters may offer an approach to rational residual stress management to enhance both the mechanical performance and service lifetime.

\section{Materials and Methods}

\subsection{Sample manufacturing and preparations}

A double tower-shaped prismatic object shown in Figure 1 with its respective dimensions was purposely designed to incorporate an abrupt change in the cross-section and the corresponding temperature and stress gradient. Samples were printed under argon atmosphere using EOS M290 SLM printer (EOS GmbH, Maisach, Germany) equipped with a $400 \mathrm{~W}$ Yb-fibre laser (IR irradiation with the wavelength of $1075 \mathrm{~nm}$ ). Powdered RS-300 alloy (contains $9.75 \% \mathrm{Si}, 0.22 \% \mathrm{Mg}, 0.2 \% \mathrm{Fe}$ ) was produced via nitrogen atomization and supplied by Valcom-PM Ltd. (Volgograd, Russia). Scanning speed used was $1200 \mathrm{~mm} / \mathrm{s}$, hatch distance $0.17 \mathrm{~mm}$ at the laser power of $370 \mathrm{~W}$. Base plate temperature was $35^{\circ} \mathrm{C}$. The layer thickness was set to $30 \mu \mathrm{m}$. "Core" parameters were applied to whole sample without any additional treatment of contours and surfaces. $10 \mathrm{~mm}$ thick support base (filling ratio $50 \%$ ) was mechanically cut. No post-printing heat treatment (aging) was applied to release residual stress. The sample was shelved for 6 months in ambient conditions and dry atmosphere before any further manipulations.
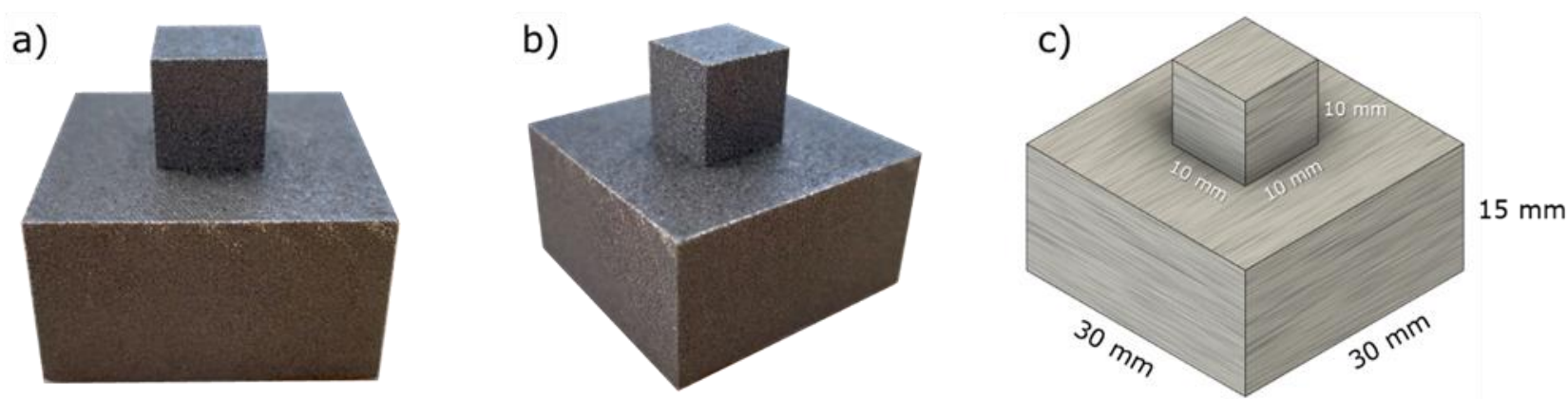

Figure 1. The appearance of double tower shape sample in the as-printed state: (a) front view, (b) side view, (c) dimensions 
A special casing was machined from a piece of hardened aluminium alloy D16 (Russian designation of AA2024) to ensure tight fixation of the double tower specimen during EDM cutting using Mitsubishi MV-1200R machine (Mitsubishi Electric Europe B.V., Ratingen, Germany) which was performed with the slowest cutting speed. This casing is demonstrated in Figure 2. The two halves of the specimens were then studied with a number of approaches: the contour method, macroscopic hole drilling with laser speckle interferometry, X-ray diffraction and Xe pFIB-DIC micro- ring-core drilling. Each of these methods is capable of evaluating the in-plane residual stresses that persist at the surface of the cut plane(s).
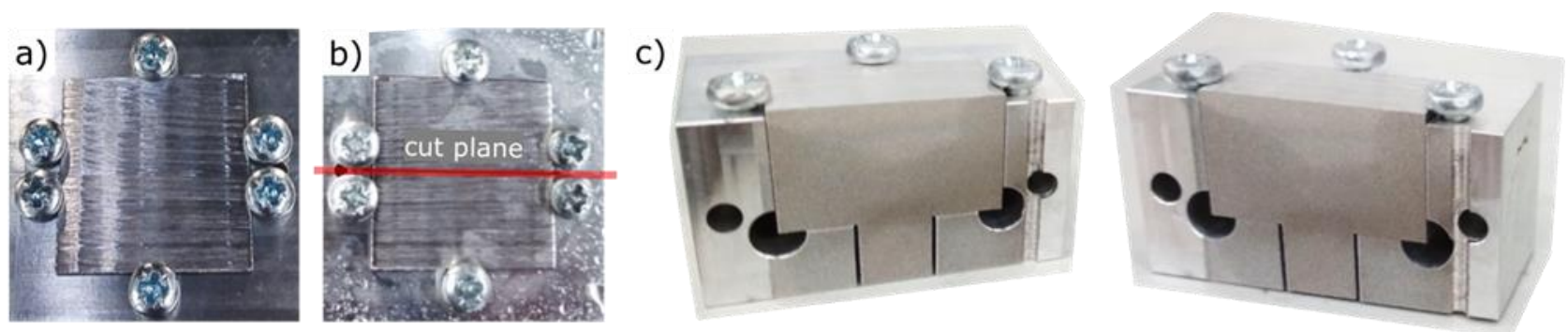

Figure 2. The appearance of the double tower sample $(\mathbf{a})$ before and $(\mathbf{b}, \mathbf{c})$ after EDM cutting: (b) top view with the indicated cut plane (red line) and (c) sectional view

\subsection{Contour method}

Optical 3D scanner RangeVision PRO Base (RangeVision Ltd., Moscow, Russia) was used to acquire volumetric profilometry data (surface point positions). This procedure was used to generate a digital geometry image (digital twin) of the double tower sample as a cloud of points before and after EDM cutting along the red line shown in Figure $2 b$. The scanner parameters were the following: working distance $260 \mathrm{~mm}, 3 \mathrm{D}$ resolution 0.05 $\mathrm{mm}$, scanning resolution $18 \mu \mathrm{m}$. 3D models before and after EDM cutting are demonstrated in Figure 3.
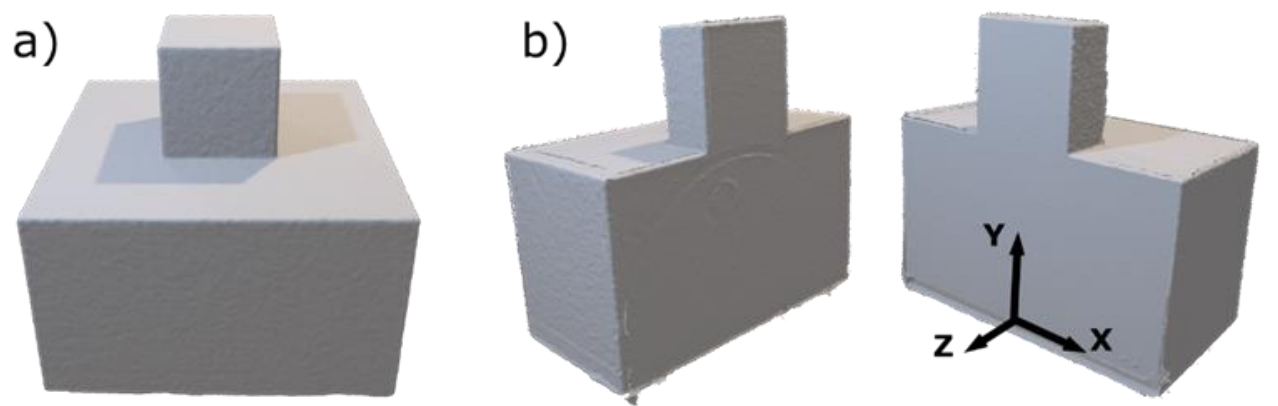

Figure 3. The appearance of double tower shaped sample as 3D models: (a) before and (b) after EDM cutting in half with the indicated primary coordinate system according to which all used methods are aligned

FEniCS which is an open-source (LGPLv3) computing platform for solving partial differential equations (PDEs) was used to refine scanned set of digital data (cloud of points) aiming to reconstruct "true" surface geometry. The experimental data was processed using the method described in the previous study by the authors [25] for the determination of the out-of-plane surface displacements as a result of stress relaxation induced by non-disturbing EDM sectioning. Following the contour method approach, the reverse of surface displacements was assigned as displacement boundary conditions of the numerical model geometry on the surface that corresponds to the EDM cut.

It is important to point out at this stage that the Contour Method was developed and validated for the calculation of residual stresses in continuously processed bodies [26]. However, the sample geometry considered in the present study had the 3D shape of a "double tower" composed of the larger and smaller area segments. This property of the 
part along with the complexity of the SLM 3D printing process means that the situation no longer corresponds to the continuously processed body assumption. Nevertheless, a numerical model was developed and applied that uses real geometry to reconstruct the residual stresses within the sectioned half of the sample. Partial validation of the approach was sought by comparison with local residual stress evaluation using FIB-DIC micro-ringcore milling method [27]. For the purpose of comparison, another numerical model was used for a continuously processed (plane strain) body of extrusion for both larger and smaller area segments.

\subsection{Hole drilling and laser speckle interferometry method}

The hole-drilling method is a destructive technique that measures local deformations in the vicinity of a drilled hole (increment or decrement of hole diameter). Drilling removes certain amount of stressed material and, as a result, causes elastic strain redistribution of the typical order of magnitude $\sim 10^{-3}$. Few millimeter large holes, therefore, generate the displacements of about few micrometers that can be measured with sufficient accuracy. Electronic laser speckle-pattern interferometry technique that was applied for this purpose visualizes the interference fringe patterns generated by in-plane displacements. The corresponding interferograms were recorded by two symmetrical side detectors (digital cameras) when a diode laser of wavelength $532 \mathrm{~nm}$ was directed at the surface containing the drilled hole. The phase shift between the two surface states before and after drilling forms alternating dark and light interference patterns known as fringes. Typical interference fringe patterns used for residual stress determination at the point of drilling are shown in Figure 4. The number of fringes is linearly related with diameter increment or decrement. These quantities are related by the multiplication factor $\frac{\lambda}{2 \sin \theta}$, where $\lambda$ is the incident illumination light wavelength, and $\theta$ is the angle between the incident and reflected beams [28]. Drilling was conducted on the cut plane as well as outer faces of the cut in half double tower specimens using the drill bit diameter of $1.9 \ldots 2.5 \mathrm{~mm}$. The drilling depth was set equal to the drill bit diameter. The values of the hole diameter increment in the principal stress directions were used as the initial experimental information for further calculation of the principal residual stress components. The typical uncertainty inherent in the determination of the maximum in-plane principal residual stress component is of the order of $5 \%$. The approach is thought to be valid for residual stress values that do not exceed $60 \%$ of the material yield stress.
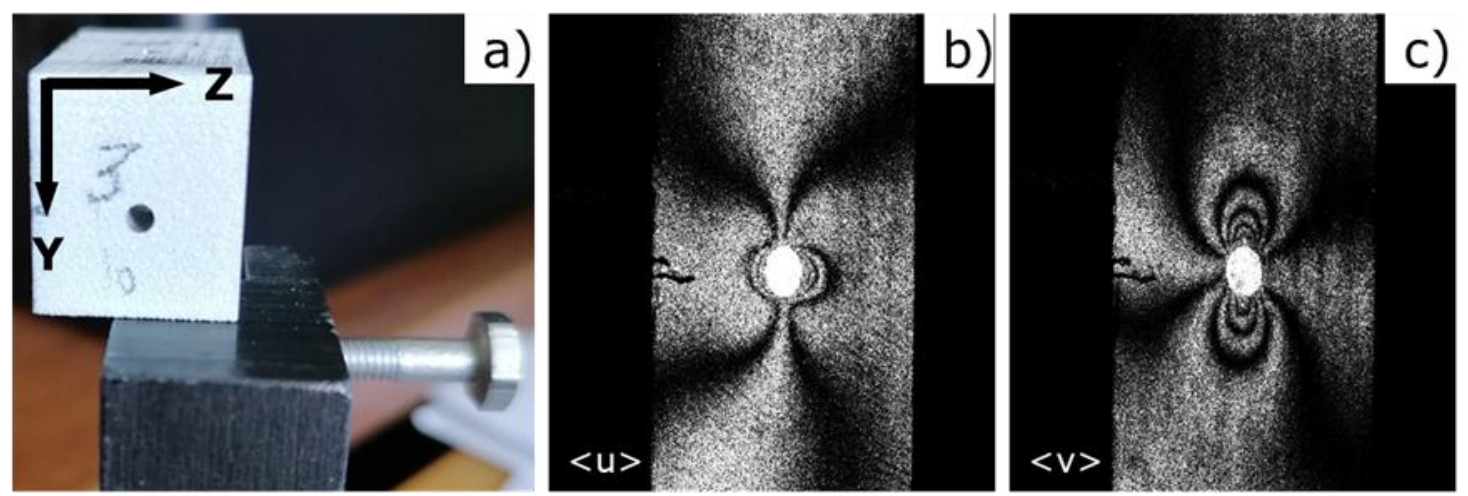

Figure 4. (a) General view of vertical outer face of the half cut double tower perpendicular to the sectioning plane with the drill hole, and $(\mathbf{b}, \mathbf{c})$ interference fringe patterns obtained as a result of $1.9 \mathrm{~mm}$ diameter hole drilling: (b) the horizontal in-plane displacement component and (c) the vertical in-plane displacement component

\subsection{X-ray diffraction method}

X-ray diffraction residual stress measurement commonly known as the $\sin ^{2} \psi$ method is widely applied and reported in the literature. Laboratory X-ray sources with $\mathrm{Cu}$ cathode generate $\sim 8 \mathrm{keV}$ characteristic radiation that probes the shallow subsurface layers to the maximum depth of $\sim 20 \mu \mathrm{m}$ for $\mathrm{Al}$ alloys. In this situation the surface roughness issue becomes a critical issue, as in the case of SLM 3D-printed parts without post- 
printing surface treatment. The typical peak-to-trough measure of roughness is believed to be comparable with the average size of the powder particles, i.e. $20 \mu \mathrm{m}$ [23]. Therefore, the cut plane of a half of the double tower samples was ground using a sequence of sandpapers in several steps corresponding to 220, 500, 1000, 2000, and 4000 grit respectively. This was followed by polishing using diamond suspensions with average particle diameter 1-3 $\mu \mathrm{m}$, and finished with colloidal silica with the average particle diameter of $60 \mathrm{~nm}$. The appearance of the cut surface after grinding and polishing is illustrated in Figure 5a. It should be noted that surface finishing by grinding and polishing may cause the re-distributing of existing and may introduce new residual stresses in the near-surface layers. However, provided good practice procedures are followed, the estimate for the thickness of the affected sub-surface layer corresponds to a few (typically, three) diameters of the final finishing silica particles [29]. That is much shallower than the penetration depth of $\mathrm{Cu}$ irradiation, so that the values of residual stress obtained with the help of X-ray diffraction can be taken as a relevant measure of the pre-existing state of the samples.

Bruker D8 Advance (Bruker AXS Inc., Madison, Wisconsin, USA) diffractometer equipped with $\mathrm{Cu}$ cathode tube $\left(K_{\alpha}\right.$ wavelength of $\left.0.154 \mathrm{~nm}\right)$ in the line collimation mode was used in combination with a special tailor-made goniometer-like sample cradle that allowed omega-tilting mode for $\sin ^{2} \psi$ analysis. The cradle was designed and made inhouse out of polylactide polymer (PLA) by FFF 3D-printing using a conventional FDM printer and PLA filament) facilitating $\omega$ (iso) and $\varphi$ (side) tilting to reach $\varphi$ values up to $60^{\circ}$ with $10^{\circ}$ steps. This holder is shown in Figure 5b. Digital X-ray diffraction spectra were processed using the conventional procedure that included fitting and subtracting the background, stripping of $K_{\beta}$ peaks, approximation of the peak profile with a Gaussian function, calculation of $d_{H K L}$ from the $2 \theta$ position of the peak center, and finally the least squares fitting of the $\varepsilon=\left(\frac{d_{H K L}}{d_{H K L}^{0}}-1\right)$ vs $\sin ^{2} \psi$ dependence with a linear function and the determination of the residual stress component from the fitted slope $\mathrm{s}$ using the X-ray Elastic Constant (XEC) of the material using

$$
\sigma_{i s o}=\mathrm{XEC} \times s=\frac{E}{(1+v)} \times \frac{d \varepsilon_{h k l}}{d\left(\sin ^{2} \psi\right)} .
$$

a)

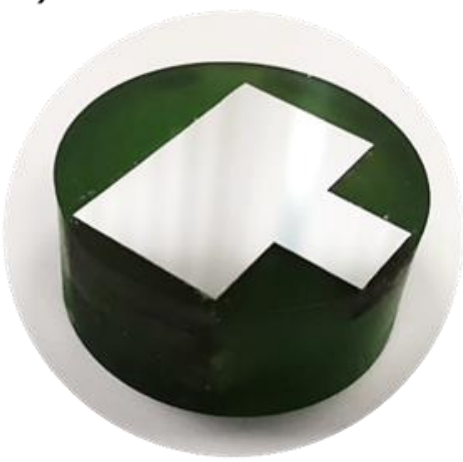

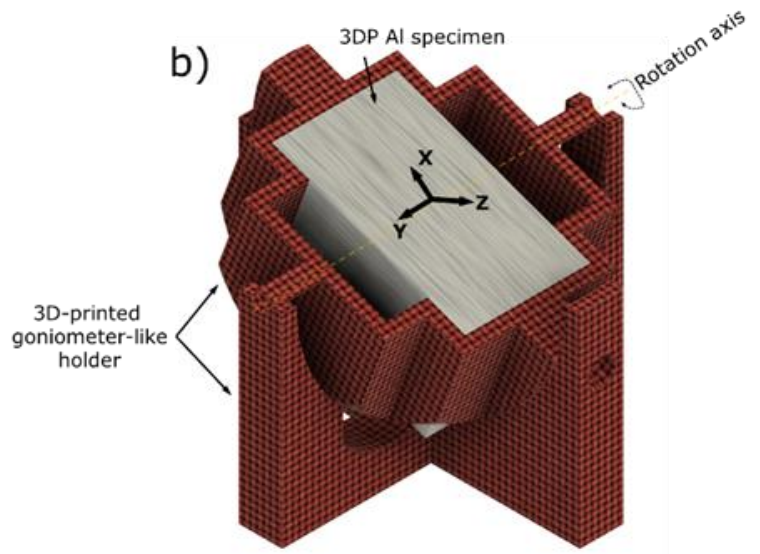

Figure 5. Appearance of the polished half cut double tower sample: (a) mounted in conductive resin for FIB-SEM studies, (b) fixed in the goniometer-like sample holder for X-ray diffraction measurements

\subsection{Xe pFIB-DIC micro-ring-core drilling method}

The semi-destructive method known as FIB-DIC micro-ring-core drilling monitors the evolving strains at the top surface of the cylindrical core during ion etching of a ringshaped trench in a FIB-SEM system. The strains are evaluated by means of digital image correlation algorithms capable of detecting the displacements of pixel clusters with subpixel accuracy. Datasets of high-resolution images are accumulated by collecting good quality SEM images after each step of serial ion milling. As a rule, the full depth of the ring trench drilled with ions lies in the range of 1-2 core diameters to guarantee the 
complete relaxation of residual stresses in the core zone. The value of the complete relief strain is used to calculate the residual stresses present at the ring-core location prior to drilling. The FIB-DIC micro-ring-core method is sensitive to a number of factors that must be considered to ensure robust residual stress evaluation [21]. Optimal density of surface features must persist at the top surface during ion ring milling without being adversely affected by the re-deposition processes or by etching by divergent ion beam.

Xe plasma FIB has been less frequently used for the residual stress analysis [30] compared to the conventional Ga FIB. A significant reason for the lower usage of Xe pFIB-SEM instruments for residual stress measurements concerns the higher cost of Xe pFIB-SEM microscopes in terms of purchase, maintenance and consumables. On the other hand, the higher plasma FIB milling currents offers the opportunity to drill larger rings with typical diameters in the range $20 \ldots 100 \mu \mathrm{m}$ in reasonable time. This offers the possibility of filling the spatial resolution gap between the Ga FIB-DIC method $(1 \ldots 20 \mu \mathrm{m})$ and mechanical drilling $(500 \ldots 3000 \mu \mathrm{m})$. It is the authors opinion that is substantiated with the results presented below that the technical difficulties related with material re-deposition can be overcome through careful optimization of milling parameters.

The present study made use of Xe pFIB-DIC micro-ring-core drilling to match the large average grain size in the material $(>20 \mu \mathrm{m})$ to fulfil the requirement of the core diameter including many or at least several grains [29]. Tescan Clara SEM equipped with Oxford Instruments NordlysNano EBSD detector was used to visualize the grain microstructure using $20 \mathrm{kV}, 30 \mathrm{nA}$ beam settings. The area of $500 \times 500 \mu \mathrm{m}^{2}$ was scanned using a $1024 \times 1024$ pixel matrix, corresponding to the scanning step of $0.5 \mu \mathrm{m}$.

Xe pFIB FEG-SEM Helios 5 microscope (FEI, Thermo Fisher Scientific Inc, USA) was used for pFIB-DIC ring-core drilling and synchronized SEM visualization using SE detector of the surface topography at the FEG acceleration voltage of $5 \mathrm{kV}$ and electron beam current of $3.2 \mathrm{nA}$. Optimal voltage and current parameters of ion milling were empirically adjusted through trial-and-error to the optimal setting of $30 \mathrm{kV}$ voltage and $200 \mathrm{nA}$ ion current. The tilt stage angle of $52^{\circ}$ was used, with the ring-core diameter of $70 \mu \mathrm{m}$ and the outer diameter of $100 \mu \mathrm{m}$. Datasets of 38 images per milling were collected, with the first 30 images corresponding to the step of $2 \mu \mathrm{m}$, and the final 7 images taken with the step of $6 \mu \mathrm{m}$, yielding the overall milling depth of $\sim 100 \mu \mathrm{m}$.

The quality of the EDM-sectioned plane provided a good density of irregular surface features. However, increased surface roughness impedes uniform ion drilling and synchronous SEM imaging due to the low visibility of the ring milling features at the $52^{\circ}$ stage tilt required for ion milling due to the high surface asperities caused by EDM cutting. On the other hand, sufficient density of contrasting surface features within the ring-core were found after silica finishing and thus provided satisfactory contrast for robust DIC analysis and was therefore used for pFIB-DIC evaluation. Open-source Matlab-based software iStress [31] was applied to evaluate the strain values in the range $10^{-4} \ldots 10^{-2}$ using image datasets of up to 60 images for the ring-core diameters of $70 \mu \mathrm{m}$.

\section{Results and discussion}

\subsection{Residual stress evaluation by the contour method}

Calculations of the linear elastic numerical model were performed by setting Young's modulus and Poisson's ratio to the values of $67 \mathrm{GPa}$ and 0.33 , respectively, in accordance with the values determined by mechanical testing of 3D-printed $\mathrm{Al}$ alloy samples.

The conventional formulation of the contour method [32] assumes that the object being considered satisfies the assumptions for the so-called continuously processed body. To rationalize these assumptions based on a firm theoretical footing it is best to state that the object finds itself in the state of plane eigenstrain. This means that the permanent inelastic strains that act as the sources of residual stress are distributed within a prismatic body of extrusion as a function of the transverse coordinates only. As a consequence, each transverse cross-section of the body finds itself in an identical state of residual stress. This, in turn, can be recovered by performing minimally disturbing planar cut e.g., using 
Electric Discharge Machining (EDM) of metallic alloy component, mapping the out-ofplane displacements (deplanation) and applying them to a numerical model as a boundary condition with the opposite sign to re-create the out-of-plane stress distribution as a contour map. Although some theoretical and practical limitations of this approach can be identified, the method has been shown to be successful, and was validated by comparison with independent measurements e.g., by neutron diffraction [33]. Another important aspect of the Contour Method concerns the reconstruction of in-plane stress components: although this consideration is not included in the original formulation by Prime [33], attempts have been made subsequently to address this important matter, e.g., by Uzun et al. using the eigenstrain approach [25]. In the discussion below, the conventional contour method treatment based on the continuously processed body (plane eigenstrain) assumption is compared and contrasted with the use of the real component geometry for the residual stress reconstruction.

Figures $6 \mathrm{a}$ and $6 \mathrm{c}$ show the z-component of residual stresses obtained for the real and continuously processed geometry models respectively, calculated using the displacements defined as boundary conditions on the EDM-cut front face of the model located at the positive $\mathrm{z}$ side. The displacements induced in the body are illustrated in Figures $6 \mathrm{~b}$ and $6 \mathrm{~d}$. On the other hand, residual stresses vanish with increasing depth along the $\mathrm{z}$-axis in the main body, in a way characteristic of the contour method [26]. The calculations that belong to the main body are consistent with the continuously processed body assumption. The stress magnitudes within the smaller area segment in the real and continuously processed body model geometries show high discrepancy, while similar distributions are observed in the larger area segments. The discussion and understanding of the mathematical implications of this difference lie outside the scope of the present study and will be examined separately.

a)

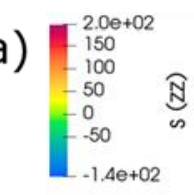

C)

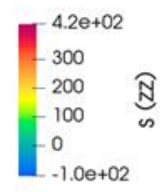

$2 x$
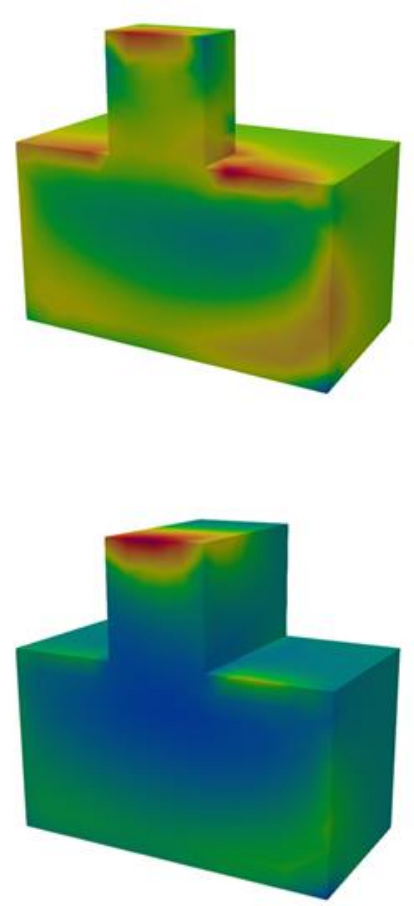

b)
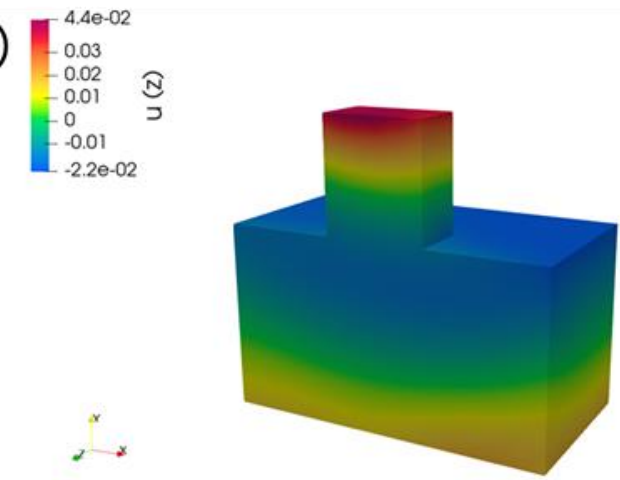

d)
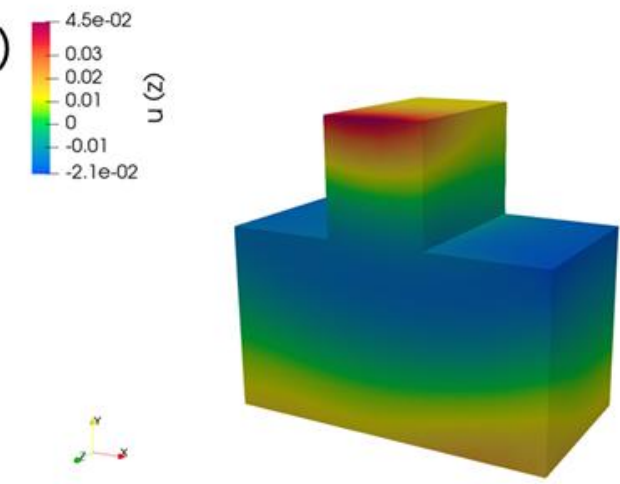

Figure 6. Illustration of the distribution of $z$-component of residual stresses $(\mathbf{a}, \mathbf{c})$ and $z$-component of displacements $(\mathbf{b}, \mathbf{d})$ in the real $(\mathbf{a}, \mathbf{b})$ and continuously processed $(\mathbf{c}, \mathbf{d})$ geometry models respectively

Although the numerical model calculates all normal and shear components of residual stress in the whole body, only z-component of the results for the "cut double tower" model geometry is reliable on the front face of the bottom segment. The results that belong to the upper segment and other components of the stress are thought to be less reliable 
but may represent a good predictive approximation. Figure 7 illustrates $\mathrm{x}$ - and $\mathrm{y}$-components of direct residual stress at the examined cut surface. Figure 8 shows all components of residual stress along the lines illustrated in Figure 7. The results show that the calculations of the contour method solution provide a good match with FIB-DIC evaluations of the x-component of residual stress in both real and continuous geometry models, but the quantity and quality of experimental measurements should be increased in order to state that all direct components of residual stress can be calculated reliably using the present approach. In addition, the comparison between the line plots of the real and continuous geometry models shows that residual stresses in the real geometry model lies within the elastic region while they extend far beyond the yield strength of the material in the continuous geometry model. Accordingly, it can be stated that the real and continuous geometry models provide similar distributions in the large section of the model that satisfies the requirements of continuous processing while they show disagreement in the small area section.

The contour method depends on the elastic response of the material subsequently to non-disturbing EDM sectioning. This relationship allows reconstructing the out-of-plane residual stress component within the section corresponding to the state of component before sectioning. On the other hand, the $\mathrm{x}$-component of residual stress evaluated using XepFIB micro-ring-core milling technique belongs to the sectioned surface. On this surface, the out-of-plane component of residual stress is completely relaxed, while the $\mathrm{x}$ - and $\mathrm{y}$ components of residual stress obtained from the Contour Method correspond to the presectioning state. It is worth noting that according to Lunt et. al. [34], sectioning involves a relief of in-plane residual stresses proportionally to $\left(1-v^{2}\right)$, where $v$ is Poisson's ratio. Based on the outputs of that study, it can be stated that the effect of sectioning on the XepFIB residual stress evaluation corresponds to $\sim 10 \%$ modification.

a)

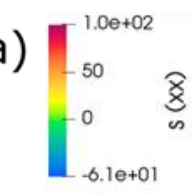

c)

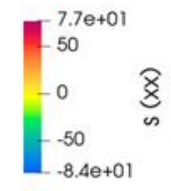

2 b)
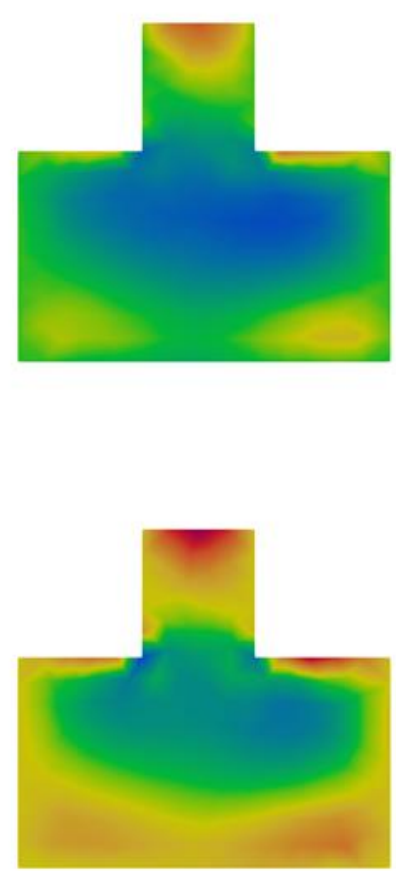

d)
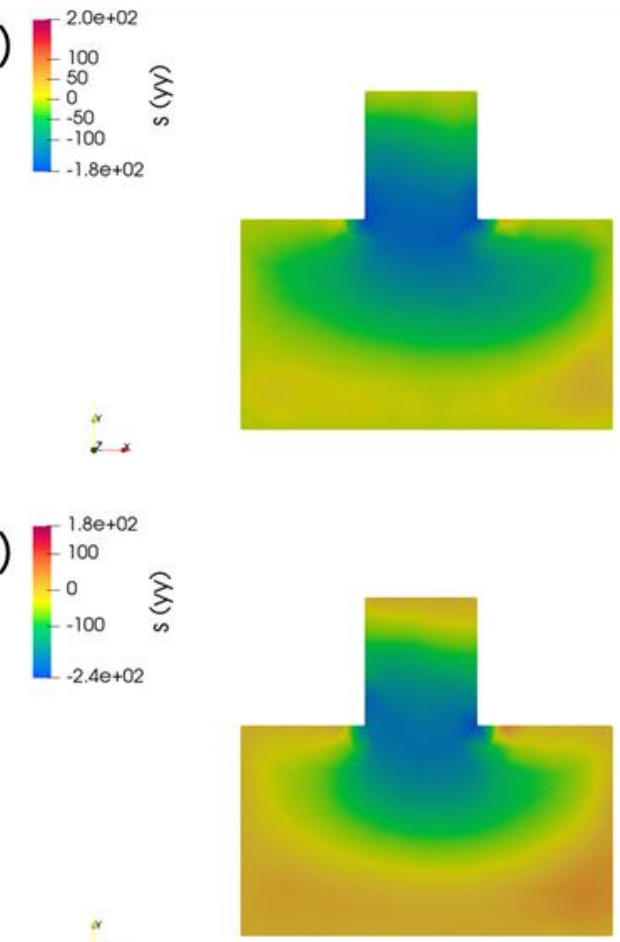

Figure 7. Illustration of distribution of $x-(\mathbf{a}, \mathbf{c})$ and $y-(\mathbf{b}, \mathbf{d})$ components of residual stresses in the real $(\mathbf{a}, \mathbf{b})$ and continuous $(\mathbf{c}, \mathbf{d})$ geometry models respectively 

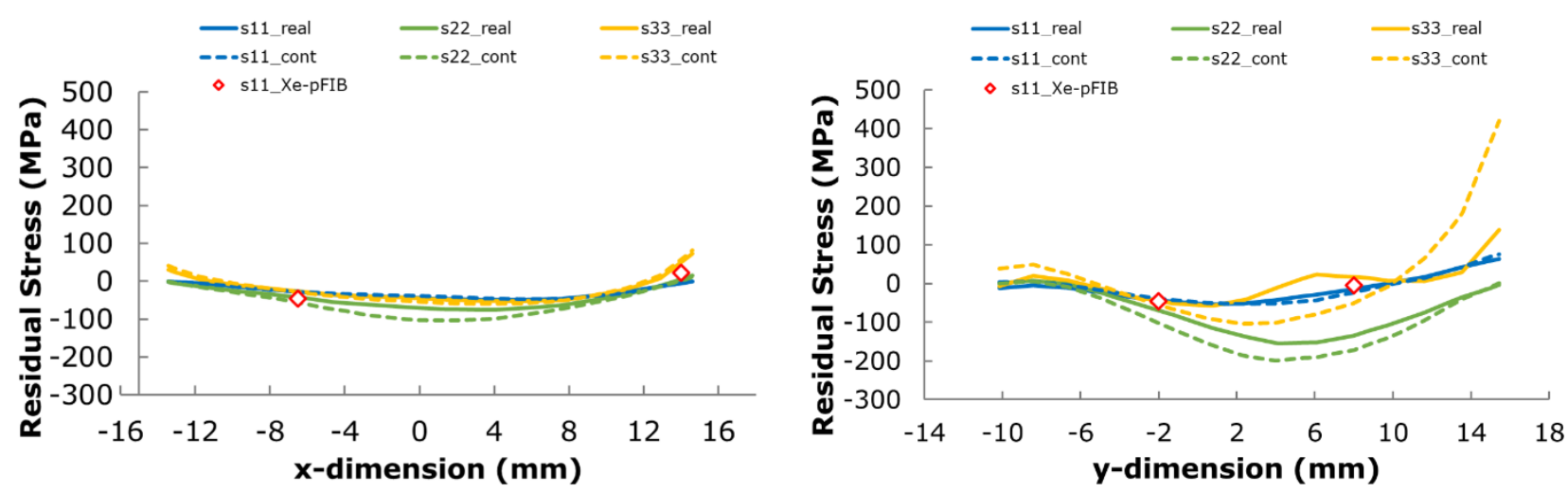

Figure 8. Line plots of $x-, y$ - and $z$-components of residual stress along the horizontal (a) and vertical (b) lines illustrated as black lines on the sectioned surface in Figure 7

The relatively high cooling rate in the top-of-tower and bottom-of-basement locations suggests fast thermal contraction in respect to the middle regions to be balanced by tensile residual stresses. This provides a well-founded explanation for the mechanical response of dog-bone samples SLM 3D-printed in different orientations under tension, and the beneficial effect of sand-blasting described in [24]. Specifically, the sample oriented identically to the bottom of the base of the double tower specimen was found to possess the lowest strength. This is likely to be linked to the inherited tensile residual stresses, but is dramatically improved in terms of ductility after sand-blasting that is known to introduce compressive residual stresses [35].

The middle part of SLM 3D-printed is relatively thermally insulated, thus, it cools and shrinks latest and, being thermally expanded because of this, inherits compressive residual stresses. Vertical outer surfaces of heavy basement adjacent to cut plane seem to be weakly stressed (small tensile or zero residual stresses), perhaps because of relatively slow cooling due to the significant thermal mass of basement - contrary to the case of tower, where temperature gradient is sharp to cause intensive heat flow (perhaps, even from basement), fast cooling and large tensile residual stresses. This observation, being quite trivial and obvious, is of, nevertheless, great practical importance suggesting that slim cross-sections and protruding elements should be repetitively printed at slower scanning speed to retain high temperature as long as possible. Annealing or shot peening postprocessing treatments are recommended to be deliberately focused on these locations.

\subsection{Residual stress evolution by hole drilling and laser speckle interferometry method}

EDM cutting of the double tower specimen causes the residual stresses to be re-distributed and relieved. Nevertheless, as discussed above, that this effect is relatively moderate in magnitude (of the order of $10 \%$ ) and takes place primarily in the vicinity of the cut.

Figure 9 demonstrates the locations of the probing holes, with the diameter of point 1 equal to $2.5 \mathrm{~mm}$, and all other points having the diameter of $1.9 \mathrm{~mm}$. The axes are designated at each probed face, with examples of interferograms with fringes shown for point 1. Table 1 summarizes the estimations of residual stresses at 6 locations where fringes were reliable detected. Four of the drilled holes revealed no fringes, indicating low magnitude of residual stresses at the probed locations. 

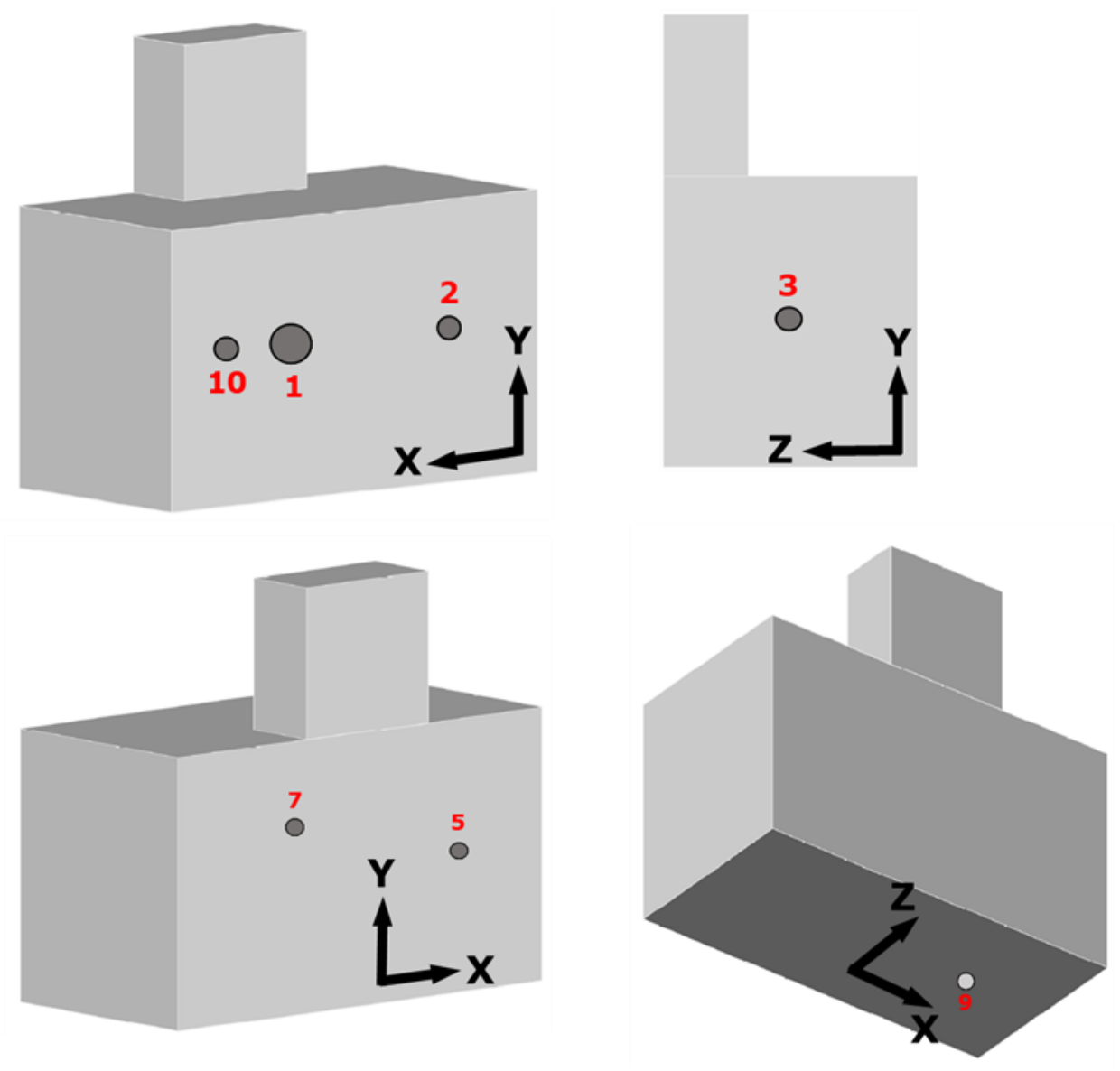

Figure 9. Locations of holes for hole drilling method with the indicated axis

Unfortunately, the size of samples was not appropriate for drilling numerous holes, as would be desirable to collect further data for accurate mapping with satisfactory spatial resolution.

Table 1. Residual stresses at half cut double tower sample faces

\begin{tabular}{|c|c|c|}
\hline $\mathbf{N}$ & Description of location & $\sigma, \mathrm{MPa}$ \\
\hline 1 & \multirow{3}{*}{ Vertical outer face parallel to the cut plane } & $120\left(\sigma_{x x}\right)$ \\
\hline 2 & & $118\left(\sigma_{x x}\right)$ \\
\hline 10 & & $129\left(\sigma_{x x}\right)$ \\
\hline 3 & Vertical outer face perpendicular to the cut plane & $-31\left(\sigma_{z z}\right)$ \\
\hline 5 & \multirow{2}{*}{ Cut plane } & $-47\left(\sigma_{x x}\right)$ \\
\hline 7 & & $-17\left(\sigma_{x x}\right)$ \\
\hline 9 & Bottom of the base & $11\left(\sigma_{x x}\right)$ \\
\hline
\end{tabular}

Despite the fact, that out-of-plane residual stresses (evaluated in contour method) are not and should not be equivalent to in-plane residual stresses in the same locations we have to notice that the middle of basement (points 5 and 7) is stressed in compression. This observation fully corresponds to the findings of contour method for out-of-plane residual stresses in the same locations. We suggest that the middle of basement is hydrostatically compressed by residual stresses due to the late cooling (and thermal expansion) to be balanced by compression. On the contrary, outer faces are residually stressed in tension as discussed above.

The components of residual stresses perpendicular to cut plane (and parallel to outof-plane residual stresses measured by contour method) at the faces adjacent to cut 
plane - points 3 and 9 - are, however, relatively small (-17 ...+11 MPa) both for vertical and horizontal bottom-of-basement faces, perhaps due to the relaxation caused by EDM cutting. Vertical outer face parallel to cut plane and far from cut plane - points 1, 2 and 10 reveals strong tensile residual stress, the values of which along horizontal axis (118...128 $\mathrm{MPa}$ ) are close to one third of yield strength of this alloy after SLM 3D-printing, that is in the range of $280 \ldots 350 \mathrm{MPa}$ [36]. Residual stresses along vertical axis are at least three times smaller suggesting uneven cooling along this face.

\subsection{Residual stress evolution by X-ray diffraction method}

The roughness $R_{a}=1.9 \mu \mathrm{m}$ and $R_{z}=11.2 \mu \mathrm{m}$ were measured by the optical profilometer of NanoScan-4D system (TISNCM, Troitsk, Russia) before the experiment. X-ray diffraction $\left(\sin ^{2} \varphi\right)$ method shows modest compressive residual stresses $(-300 \ldots-350 \mathrm{MPa})$ in the cut plane as shown in Figure 10. This method measures in-plane residual stresses only and, what is also very important, assumes that all grain subsets (and all grain in a subset) diffracting at different $\varphi$ angles are evenly and equally stressed as those grain subsets having $(H K L)$ planes perpendicular to the cut plane $\left(\sin ^{2} \varphi=1\right)$. Averaging over lighted area (from few tens to few hundreds of $\mathrm{mm}^{2}$ typical for laboratory X-ray) in the middle of cut plane gives no spatial resolution, which potentially can be improved with dedicated setups utilizing fine focus tubes and advanced sample positioning systems. We consider these results as elective.
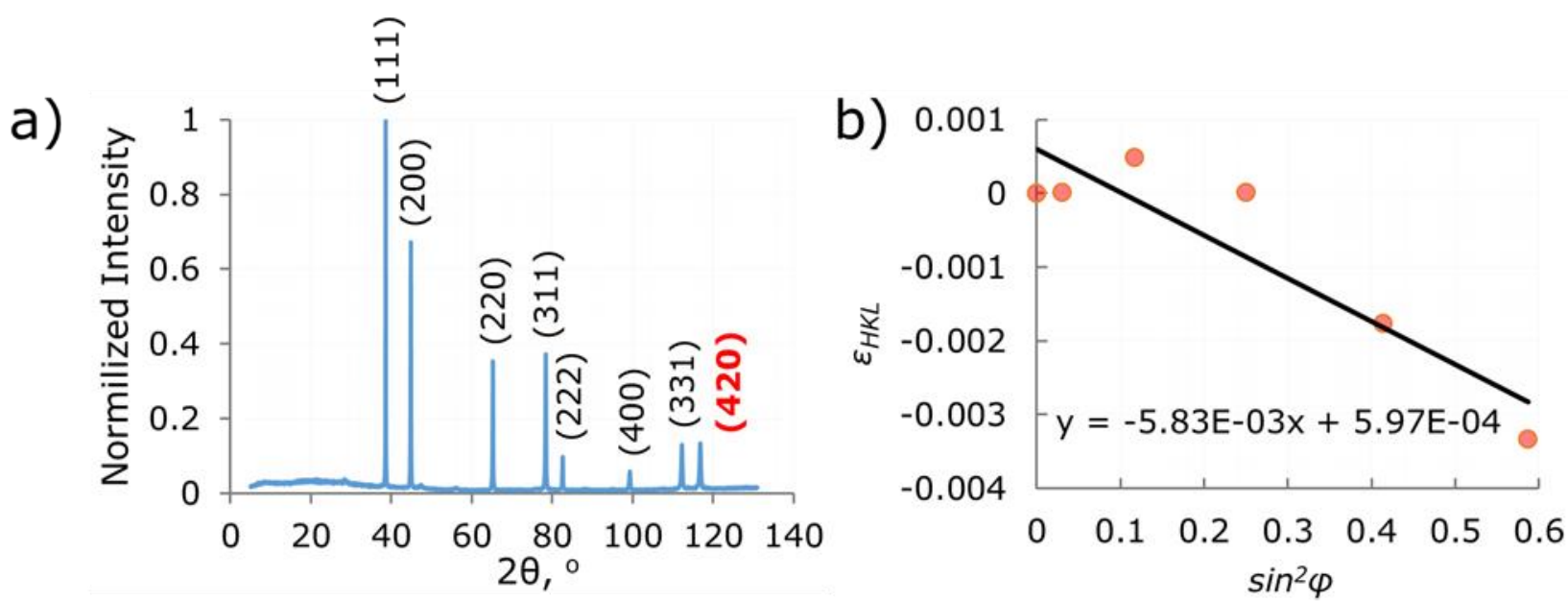

Figure 10. Details of $X$-ray measurement of residual stresses at the cut plane: (a) measured $I \sim 2 \theta$ plot for the specimen (highlighted red plane (420) was selected for the next calculations); (b) $\varepsilon_{H K L} \sim \sin ^{2} \varphi$ plot

\subsection{Residual stress evolution by Xe pFIB ring-core drilling method}

Figure 11 depicts typical appearance of ion drilled ring-core holes at the rough and polished surfaces of cut plane at different half cut double tower specimens. Figure12 maps residual stresses along $\mathrm{x}$ axis in 6 points at polished cut plane and the values of evaluated in-plane residual stresses are in satisfactory agreement with the results obtained for outof-plane residual stresses (contour method). Stress profile along line $\mathrm{H} 1-\mathrm{H} 2-\mathrm{H} 3$ corresponds to the change of residual stresses from strong tensile (exceeding yield strength level) at the top of tower to modest compressive (about $1 / 3$ of yield strength). Stress profile along line $\mathrm{H} 5-\mathrm{H} 3-\mathrm{H} 4$ demonstrates weak tensile residual stresses close to vertical faces and somewhat stronger compressive residual stresses in the middle of cut plane. Xe-pFIB ring-core drilling method returns the estimation of residual stresses in location $\mathrm{H6}$, which contradicts to contour method and requires further deeper investigations. Taking into account that outer diameter of ring hole is $100 \mu \mathrm{m}$ and may be easily reduced down to 20 $\mu \mathrm{m}$ one can expect that accessible spatial resolution of residual stresses varies over the range of $60 \ldots 300 \mu \mathrm{m}$ (three ring hole diameter). That is attractive simultaneously for fundamental scientific research purposes and practical engineering tasks, since $100 \mu \mathrm{m}$ scale Xe-pFIB drilling fills the gap between mechanical drilling with $0.5 \mathrm{~mm}$ drills and Ga-FIB 
drilling with maximal rational ring holes of $20 \mu \mathrm{m}$ diameter. Economical aspects must, therefore, be carefully assessed before the conduction of high-resolution FIB ring-core drilling mapping of residual stresses.
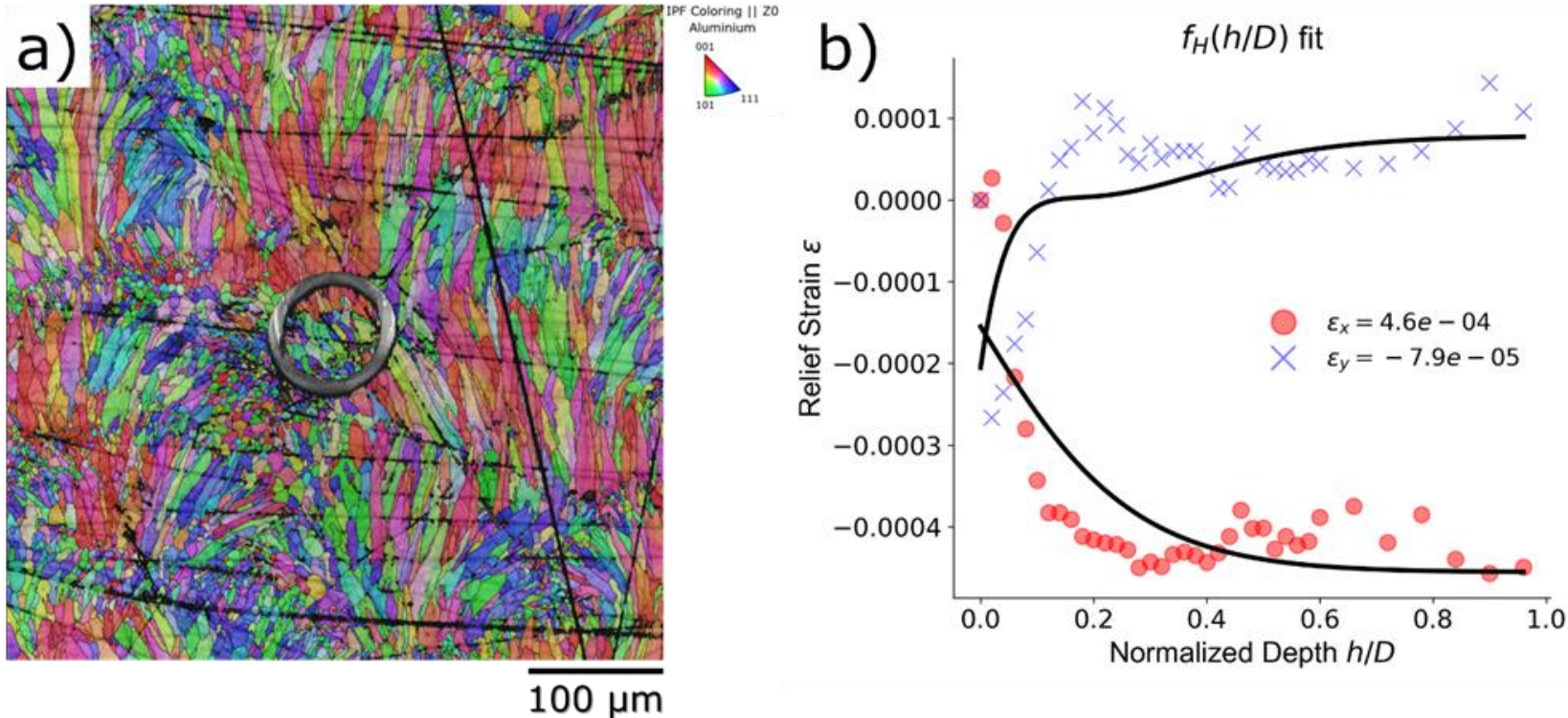

Figure 11. Details of Xe-pFIB ring-core drilling measurement of residual stresses at the cut plane: (a) combined EBSD map with the Euler' colours and milled ring and (b) measured relief strains with the fitting curve
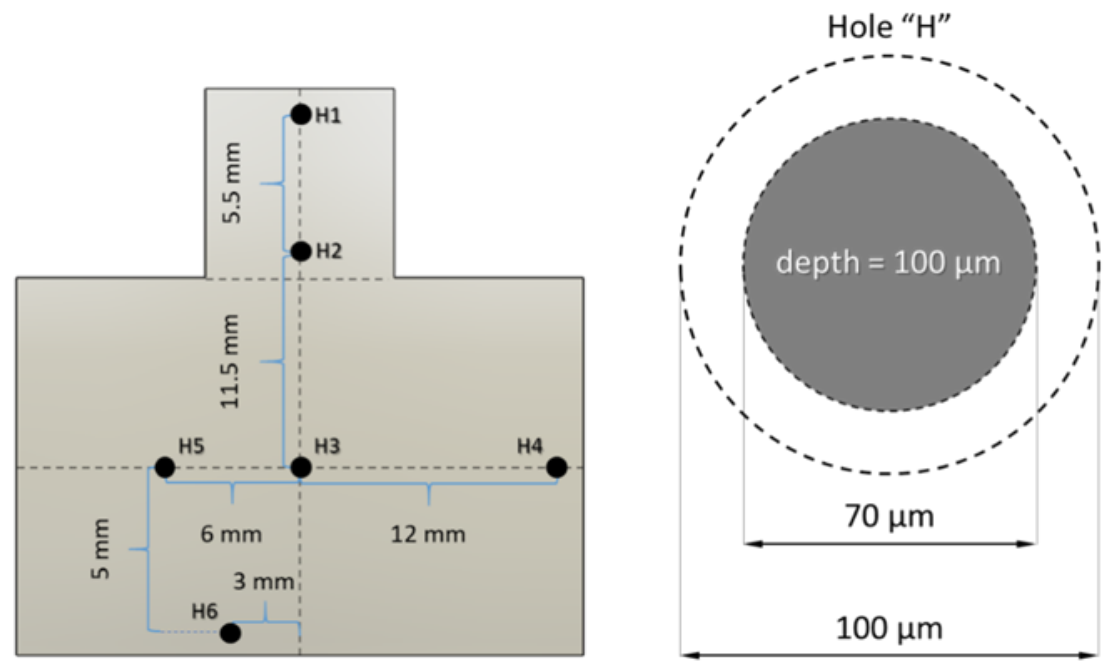

Figure 12. Mapping of residual stresses using Xe-pFIB ring-core drilling method

Table 2. Residual stresses at faces and cut plane of half cut double tower specimens

\begin{tabular}{cc}
\hline $\mathbf{N}$ & $\boldsymbol{\sigma}_{\boldsymbol{x} x}, \mathbf{M P a}$ \\
\hline $\mathrm{H} 1$ & 62.1 \\
\hline $\mathrm{H} 2$ & -4.9 \\
\hline $\mathrm{H} 3$ & -45.8 \\
\hline $\mathrm{H} 4$ & 33.4 \\
\hline $\mathrm{H} 5$ & 22.8 \\
\hline $\mathrm{H} 6$ & -78.7 \\
\hline
\end{tabular}




\section{Conclusions}

Figure 13 shows comparison plot of all used methods. The results show that the calculations of the contour method solution provide a good match with FIB-DIC and hole drilling evaluations of the $x$-component of residual stress, but the quantity and quality of experimental measurements should be increased in order to state that all direct components of residual stress can be calculated reliably using the present approach.

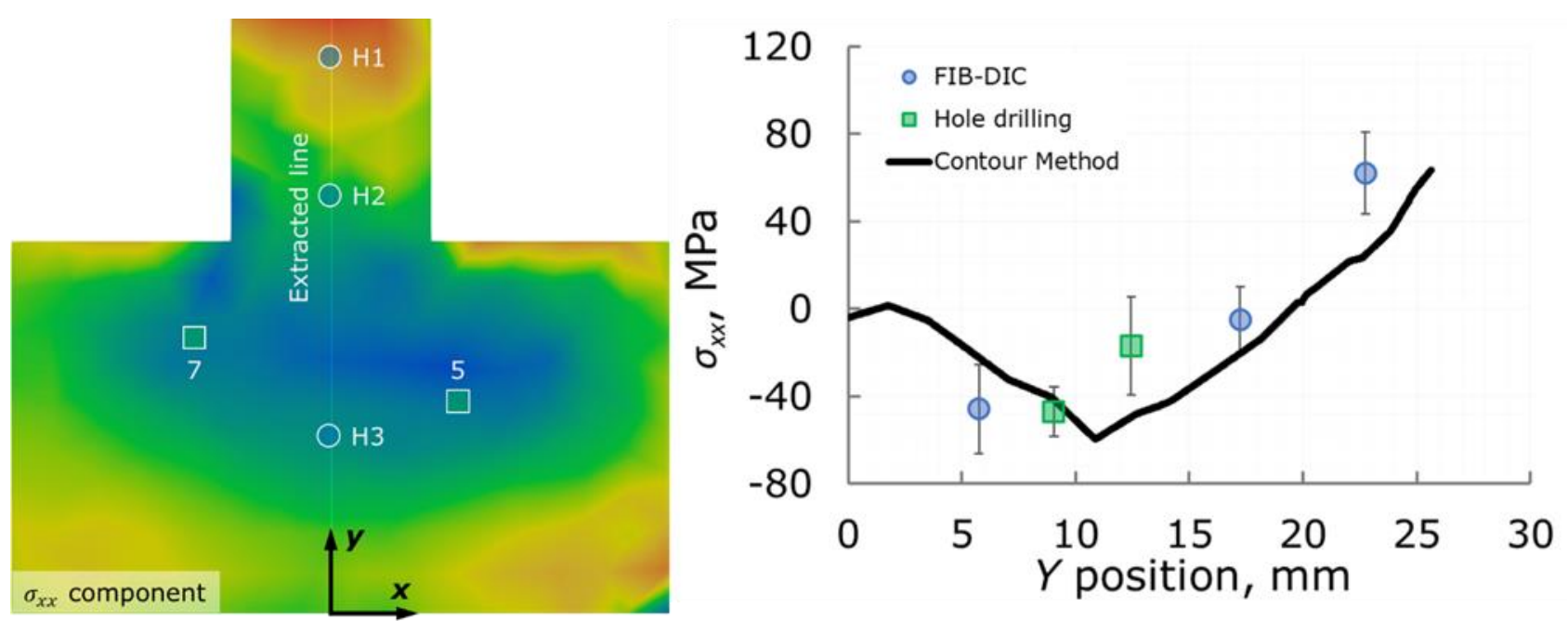

Figure 13. Comparison plot of all used methods

To sum up, residual stresses in SLM 3D-printed metal parts appearing as a result of complex thermal history are frequently discussed in respect of many aspects of their mechanical performance and operation service life. Although recovery annealing or various surface treatments (sand blasting, shot peening etc.) are readily applicable the efficiency of these measures is hardly to be assessed without relevant and robust technique for routine experimental measurements. Popular X-ray diffraction method, especially laboratory, has a number of disadvantages and shallow probing and averaging over large lighted areas are the most obvious of them.

By today growing number of experimental investigations is still based on X-ray diffraction measurements what deprives researchers much valuable information. SLM 3Dprintable Al-Si-Mg alloys seem to the least studied in comparison with printable steels, $\mathrm{Ti}$ and Ni alloys.

We deliberately undertook comparative residual stress evaluation using methods of contour measurements, mechanical drilling with speckle-pattern interferometry, X-ray diffraction and Xe-pFIB ring-core drilling with DIC analysis. These destructive and nondestructive methods have spatial resolution from tens of millimeters for contour measurements to units of millimeters for speckle-pattern interferometry and finally down to 70 micrometers for Xe-pFIB ring-core drilling. All methods returned the estimations of residual stresses occurring in good agreement in terms of signs and values regardless dramatically different spatial resolution.

The surface of SLM 3D-printed parts is residually extended with stresses having values equal and exceeding yield strength at the locations where fastest cooling takes place - protrusions, slim cross-sections and supports. The middle parts of large cross-section are residually compressed (perhaps hydrostatically) with relatively weak stresses of about $1 / 4 \ldots 1 / 3$ of yield strength.

We believe that these findings rationally explain the anisotropy of mechanical response for the parts printed with different orientations in respect to fast scanning and growth direction.

Author Contributions: Conceptualization, A.M.K., A.I.S. and D.K.R.; methodology, E.S.S., S.A.L., S.I.E., A.G.S., Yu.V.M. and P.A.S.; software, E.S.S., F.U. and S.I.E.; validation, E.S.S. and F.U.; formal analysis, A.I.S. and E.S.S.; investigation, E.S.S., S.A.L. and F.U.; resources, A.I.S., E.S.S., F.U. and 
A.M.K.; data curation, E.S.S.; writing - original draft preparation, A.I.S. and E.S.S.; writing - review and editing, A.M.K.; visualization, E.S.S.; supervision, A.M.K. and A.I.S.; project administration, A.M.K. and A.I.S.; funding acquisition, A.M.K. and A.I.S. All authors have read and agreed to the published version of the manuscript.

Funding: This research was funded by the Russian Science Foundation, grant number 21-19-00791, https://rscf.ru/en/project/21-19-00791/.

Data Availability Statement: In this section, please provide details regarding where data supporting reported results can be found, including links to publicly archived datasets analyzed or generated during the study. Please refer to suggested Data Availability Statements in section "MDPI Research Data Policies" at https://www.mdpi.com/ethics. You might choose to exclude this statement if the study did not report any data.

Acknowledgments: This research was funded by the Russian Science Foundation, grant number 21-19-00791, https://rscf.ru/en/project/21-19-00791/. The authors are grateful to the crew of the Fablab (Skoltech, Moscow, Russia) for their delicate and accurate sample preparations and Andrey Dyakov from CDMM (Skoltech, Moscow, Russia) facilitated optical 3D scanning.

Conflicts of Interest: The authors declare no conflict of interest.

\section{References}

1. Barriobero-Vila P., Gussone J., Stark A. et al. Peritectic Titanium Alloys for 3D Printing. Nat. Commun. 2018, 9.

2. Nesma T. Aboulkhair, Marco Simonelli, Luke Parry, Ian Ashcroft, Christopher Tuck, Richard Hague. 3D Printing of Aluminium Alloys: Additive Manufacturing of Aluminium Alloys using Selective Laser Melting. Progress in Materials Science $2019,106$.

3. Liu Zhiying, He Bei, Lyu Tianyi, Zou Yu. A Review on Additive Manufacturing of Titanium Alloys for Aerospace Applications: Directed Energy Deposition and Beyond Ti-6Al-4V. Journal of the Minerals 2021, 73(6).

4. Webster G.A., Ezeilo A.N. Residual Stress Distributions and Their Influence on Fatigue Lifetimes. International Journal of Fatigue 2001, 23.

5. Tim J. Carter. Common Failures in Gas Turbine Blades. Engineering Failure Analysis 2005, 12(2), $237-247$.

6. Uwe Zerbst, Giovanni Bruno, Jean-Yves Buffiere, et al. Damage Tolerant Design of Additively Manufactured Metallic Components Subjected to Cyclic Loading: State of the Art and Challenges. Progress in Materials Science 2021, 121.

7. Xu Song, Wei Zhai, Rui Huang, Jin Fu. Metal-Based 3D-Printed Micro Parts \& Structures. Reference Module in Materials Science and Materials Engineering 2020.

8. Alexander M Korsunsky, Gabriel M. Regino, D. Nowell. Variational Eigenstrain Analysis of Residual Stresses in a Welded Plate. International Journal of Solids and Structures 2007, 44(13), 4574-4591.

9. Casavola C., Pappalettere C., Tursi F. Residual Stress on AISI 300 Sintered Materials. Conference Proceedings of the Society for Experimental Mechanics Series 2011, 6, 201-208.

10. Bartlett J.L., Li X. An Overview of Residual Stresses in Metal Powder Bed Fusion. Additive Manufacturing 2019, 27, 131-149.

11. Lopez C., Elias-Zuniga A., Jimenez I., Martinez-Romero O., Siller H.R., Diabb J.M. Experimental Determination of Residual Stresses Generated by Single Point Incremental Forming of AlSi10Mg Sheets Produced using SLM Additive Manufacturing Process. Materials 2018, 11(12).

12. Karolus M., Maszybrocka J., Stwora A., Skrabalak G. Residual Stresses of AlSi10Mg Fabricated by Selective Laser Melting. Archives of Metallurgy and Materials 2019, 64(3), 1011-1016.

13. Colombo C., Biffi C.A., Fiocchi J., Tuissi A., Vergani L.M. Effect of Optimized Heat Treatments on the Tensile Behavior and Residual Stresses of Selective Laser Melted AlSi10Mg Samples. Key Engineering Materials 2019, 813, 364-369.

14. Xing X., Duan X., Sun X., Gong H., Wang L., Jiang F. Modification of Residual Stresses in Laser Additive Manufactured AlSi10Mg Specimens using an Ultrasonic Peening Technique. Materials 2019, 12(3).

15. Di Giovanni M.T., de Menezes J.T.O., Bolelli G., Cerri E., Castrodeza E.M. Fatigue Crack Growth Behavior of a Selective Laser Melted AlSi10Mg. Engineering Fracture Mechanics 2019, 217. 
16. Mfusi B.J., Mathe N.R., Tshabalala L.C., Popoola P.A.I. The Effect of Stress Relief on the Mechanical and Fatigue Properties of Additively Manufactured AlSi10Mg Parts. Metals 2019, 9(11).

17. Song X., Feih S., Zhai W., Sun C.N., Li F., Maiti R., Wei J., Yang Y., Oancea V., Romano Brandt L., Korsunsky A.M. Advances in Additive Manufacturing Process Simulation: Residual Stresses and Distortion Predictions in Complex Metallic Components. Materials and Design 2020, 193.

18. Beretta S., Gargourimotlagh M., Foletti S., du Plessis A., Riccio M. Fatigue Strength Assessment of "as Built" AlSi10Mg Manufactured by SLM with Different Build Orientations. International Journal of Fatigue 2020, 139.

19. Amir B., Grinberg E., Gale, Y. Sadot, O. Samuha, S. Influences of Platform Heating and Post-processing Stress Relief Treatment on the Mechanical Properties and Microstructure of Selective-Laser-Melted AlSi10Mg Alloys. Materials Science and Engineering A 2021, 822.

20. Bhaduri D., Ghara T., Penchev P., Paul S., Pruncu C.I., Dimov S., Morgan D. Pulsed Laser Polishing of Selective Laser Melted Aluminium Alloy Parts. Applied Surface Science 2021, 558.

21. Alexander M. Korsunsky, Marco Sebastiani, Edoardo Bemporad, Focused Ion Beam Ring Drilling for Residual Stress Evaluation. Materials Letters 2009, 63(22), 1961-1963.

22. Lord Jerry, Cox David, Ratzke Agnieszka, Sebastiani Marco, Korsunsky Alexander, Mughal Muhammad, Salvati Enrico. A Good Practice Guide for Measuring Residual Stresses using FIB-DIC. National Physical Laboratory (Teddington, UK) 2018.

23. Statnik, E.S.; Nyaza, K.V.; Salimon, A.I.; Ryabov, D.; Korsunsky, A.M. In Situ SEM Study of the Micro-Mechanical Behaviour of 3D-Printed Aluminium Alloy. Technologies 2021, 9, 21.

24. Somov, P.A.; Statnik, E.S.; Malakhova, Y.V.; Nyaza, K.V.; Salimon, A.I.; Ryabov, D.K.; Korsunsky, A.M. On the Grain Microstructure-Mechanical Properties Relationships in Aluminium Alloy Parts Fabricated by Laser Powder Bed Fusion. Metals 2021, 11,1175 .

25. Uzun F., Korsunsky A.M. On the Identification of Eigenstrain Sources of Welding Residual Stress in Bead-on-plate Inconel 740H Specimens. Int. J. Mech. Sci. 2018. 145, 231-245.

26. Kartal M.E., Kang Y.H., Korsunsky A.M., Cocks A.C.F.F., Bouchard J.P. The Influence of Welding Procedure and Plate Geometry on Residual Stresses in Thick Components. Int. J. Solids Struct. 2016. 80, 420-429.

27. Marco Sebastiani, Tan Sui, Alexander M. Korsunsky. Residual Stress Evaluation at the Micro- and Nano-scale: Recent Advancements of Measurement Techniques, Validation through Modelling, and Future Challenges. Materials E Design 2017, 118, $204-206$.

28. V.S. Pisarev, I.N. Odintsev, S.I. Eleonsky, A.A. Apalkov. Residual Stress Determination by Optical Interferometric Measurements of Hole Diameter Increments. Opt. and Las. in Eng. 2018, 110, 437-456.

29. Everaerts J., Salvati E., Uzun F., Romano Brandt L., Zhang H., Korsunsky A.M. Separating Macro- (Type I) and Micro- (Type II+III) Residual Stresses by Ring-Core FIB-DIC Milling and Eigenstrain Modelling of a Plastically Bent Titanium Alloy Bar. Acta. Mater. 2018; 156, 43-51.

30. Bart Winiarski, C. Rue, Philip J. Withers. Plasma FIB Spin Milling for 3D Residual Stress Measurements. Microscopy and Microanalysis 2019, 25(S2), 882-883.

31. Melanie Senn. Digital Image Correlation and Tracking (https://www.mathworks.com/matlabcentral/fileexchange/50994-digitalimage-correlation-and-tracking), MATLAB Central File Exchange. Retrieved November 30, 2021.

32. M.J. Roy, N. Stoyanov, R.J. Moat, P.J. Withers. pyCM: An Open-Source Computational Framework for Residual Stress Analysis Employing the Contour Method. SoftwareX 2020, 11.

33. Smith M., Levesque J.B., Bichler L. Residual stress analysis in linear friction welded in-service Inconel 718 superalloy via neutron diffraction and contour method approaches. Mater. Sci. Eng. 2017, 691, 168-179

34. Lunt A.J.G., Salvati E., Ma L., Dolbyna I.P., Neo T.K., Korsunsky A.M. Full in-plane Strain Tensor Analysis using the Microscale Ring-Core FIB Milling and DIC Approach. J. Mech. Phys. Solids 2016, 94, 47-67.

35. Abboud E., Attia Helmi, Shi Bin, et al. Residual Stresses and Surface Integrity of Ti-alloys During Finish Turning - Guidelines for Compressive Residual Stresses. Procedia CIRP 2016, 45, 55-58. 
36. Nesma T. Aboulkhair, Ian Maskery, Chris Tuck, Ian Ashcroft, Nicola M. Everitt. The Microstructure and Mechanical Properties of Selectively Laser Melted AlSi10Mg: The Effect of a Conventional T6-like Heat Treatment. Materials Science E Engineering A 2016, 667, 139-146. 